ESAIM: PROCEEDINGS, July 2007, Vol.18, 143-152

Jean-Frédéric Gerbeau \& Stéphane Labbé, Editors

\title{
UN MODÈLE DE MOUVEMENTS DE FOULE
}

\author{
Bertrand MAURY ${ }^{1}$ AND JuLiette VENEL ${ }^{1}$
}

\begin{abstract}
We propose a deterministic model for crowd motion, based on a Lagrangian approach: each person is taken into account individually. We are especially interested in the modelling of evacuation: people are willing to exit a room with obstacles. The model takes the form of an evolution equation which involves a multivalued operator, which is not in general maximal monotone. Using recent results on the sweeping process, we establish the well-posedness of this problem under reasonable assumptions. We propose a numerical scheme, which we apply to two realistic situations.

Résumé. Nous proposons un modèle déterministe de mouvements de foule basé sur une approche Lagrangienne où chaque individu est pris en compte. Nous nous intéressons ici à la modélisation de situations d'évacuation : des personnes veulent quitter une salle pouvant contenir des obstacles (tables, piliers). Nous aboutissons à une équation d'évolution sur la position où intervient un opérateur multivalué. La difficulté provient du fait que cet opérateur n'est pas en général maximal monotone. À partir de résultats récents sur le processus de rafle, nous établissons le caractère bien posé du problème. Nous présentons un schéma numérique que nous appliquons à la simulation de deux situations réalistes.
\end{abstract}

\section{Présentation du modèle}

Nous proposons un modèle de mouvements de foule dans lequel les personnes sont assimilées à des disques rigides, évoluant dans un espace bidimensionnel susceptible de contenir des obstacles. Ces disques ont pour rayons $r_{1}, r_{2}, \ldots, r_{n}$ et sont repérés par les coordonnées de leurs centres (voir figure 1). Ainsi la disposition des différentes personnes est donnée par le vecteur $\mathbf{q}=\left(\mathbf{q}_{1}, \mathbf{q}_{2}, . ., \mathbf{q}_{N}\right) \in \mathbb{R}^{2 N}$, espace qui sera muni de la norme euclidienne. L'approche que nous présentons ici repose sur deux principes. D'une part, chaque personne a une vitesse souhaitée, vitesse qu'elle aurait en l'absence des autres. Cette vitesse est déterminée dans le cadre d'une stratégie individuelle d'évacuation : chaque individu tend à se rendre le plus vite possible vers la sortie en prenant en compte les obstacles visibles. D'autre part, le déplacement effectif des individus doit respecter une contrainte d'encombrement maximal : les personnes ne peuvent pas se chevaucher ou traverser les obstacles. Leur vitesse réelle est donc différente et prend en compte ces contraintes. Dans notre modèle, la vitesse réelle s'écrit comme la projection euclidienne de la vitesse souhaitée sur l'ensemble des vitesses admissibles. Pour ne pas alourdir les notations, nous ne présenterons ici que le premier type de contraintes (non chevauchement des disques), étant entendu que les contraintes liées aux obstacles passifs peuvent être gérées de la même manière. On introduit pour cela l'espace des configurations admissibles, auquel doit appartenir le vecteur position $\mathbf{q}$ :

$$
Q_{0}=\left\{\mathbf{q} \in \mathbb{R}^{2 N}, \forall i, j \quad i \neq j \quad D_{i j}(\mathbf{q})=\left|\mathbf{q}_{i}-\mathbf{q}_{j}\right|-r_{i}-r_{j} \geq 0\right\} .
$$

\footnotetext{
${ }^{1}$ Laboratoire de Mathématiques, Université Paris XI, Orsay, e-mail: prénom.nom@math.u-psud.fr
} 


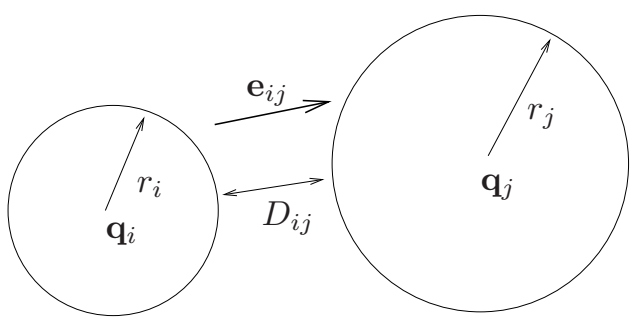

FiguRE 1. Notations

Nous allons maintenant apporter quelques précisions sur la vitesse souhaitée et la vitesse réelle.

Vitesse Souhaitée. Dans un premier temps, nous allons décrire la vitesse souhaitée d'une personne quelconque dans la foule, vitesse qui ne dépendra que de sa propre position. On définit donc en tout point $\mathrm{M}$ de la salle une vitesse souhaitée $\mathbf{U}_{0}(\mathrm{M})$, vitesse qu'aurait une personne seule, à cette position, désirant gagner la sortie. Cette vitesse est choisie en fonction de la géométrie de la pièce : un individu se déplaçant avec cette vitesse tente de minimiser la distance à parcourir pour atteindre la sortie. Le choix de cette vitesse souhaitée n'est pas unique. Une manière naturelle de la définir, qui correspond au choix effectué dans les exemples numériques de la section 4, est de considérer un champ de module constant (vitesse maximale d'un individu) dont le flot suit les plus courts chemins entre un point courant du domaine et un point à l'extérieur du bâtiment à évacuer. La figure 2 représente une salle contenant des obstacles et le flot du champ des vitesses souhaitées associé, calculé selon ce principe de plus court chemin (sauf au niveau du couloir entre les deux salles). Ce cas de figure correspond à la situation modélisée dans la section 4.2 .

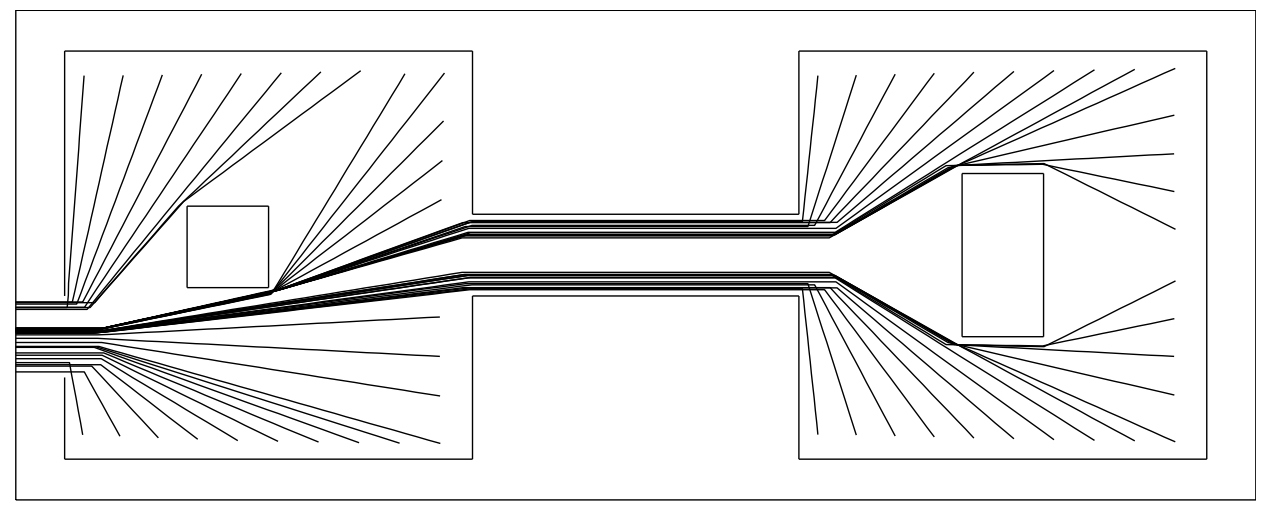

Figure 2. Flot du champ des vitesses souhaitées

En supposant que chaque individu a le même comportement, caractérisé par la vitesse $\mathbf{U}_{0}$ décrite précédemment, nous pouvons noter $\mathbf{U}(\mathbf{q})=\left(\mathbf{U}_{0}\left(\mathbf{q}_{1}\right), . ., \mathbf{U}_{0}\left(\mathbf{q}_{N}\right)\right)$ le vecteur des vitesses souhaitées des $\mathrm{N}$ personnes. Nous nous sommes limités ici au cas où la vitesse souhaitée d'un individu ne dépend pas de sa position relative vis-àvis d'autres personnes, ce qui correspond d'une certaine manière à une situation de panique dans laquelle les individus ne sont pas en mesure d'élaborer des stratégies évoluées d'évacuation qui prendraient en compte le comportement de leurs semblables.

Remarque 1. Ces stratégies plus évoluées sont susceptibles d'être intégrées à des versions futures de ce modèle. En effet, nous pourrions personnaliser la vitesse souhaitée en prenant en compte le comportement ou la capacité 
physique de chacun : les personnes peuvent courir à des allures différentes et avoir une stratégie propre pour atteindre la sortie le plus rapidement possible. Si l'on prend l'exemple de la figure 2, certaines personnes arrivant de la deuxième salle et s'apercevant d'un bouchon au niveau de l'obstacle carré pourraient choisir de l'éviter différemment, en passant, soit de l'autre côté (à droite), soit du même côté mais en contournant aussi la foule bloquée. Ainsi, le vecteur vitesse des $N$ personnes s'écrirait: $\mathbf{U}(\mathbf{q})=\left(\mathbf{U}_{1}(\mathbf{q}), . ., \mathbf{U}_{N}(\mathbf{q})\right)$, les champs $\mathbf{U}_{i}$ pouvant se distinguer par leur module et leur direction.

En ce qui concerne la régularité de $\mathbf{U}$, le caractère bien posé du problème ne pourra être établi que sous l'hypothèse que $\mathbf{U}$ est lispschitzienne par rapport à $\mathbf{q}$. Pour les situations réalistes, et notamment dès que le domaine à évacuer n'est plus simplement connexe, ce caractère lipschitzien n'est pas assuré. Ainsi le champ représenté à la figure 2 est singulier au voisinage de lignes de séparations en amont des obstacles à contourner. Nous verrons que ce problème ne pose pas de difficulté particulière pour ce qui est de la résolution numérique.

Vitesse Réelle. Pour empêcher le chevauchement des disques, on impose à la vitesse réelle notée $\mathbf{u}$ d'appartenir à $C_{\mathbf{q}}$, cône des vitesses admissibles défini par

$$
C_{\mathbf{q}}=\left\{\mathbf{v} \in \mathbb{R}^{2 N}, \quad D_{i j}(\mathbf{q})=0 \quad \Rightarrow \quad \mathbf{G}_{i j}(\mathbf{q}) \cdot \mathbf{v} \geq 0 \quad \forall i<j\right\}
$$

avec $\mathbf{G}_{i j}(\mathbf{q})=\nabla D_{i j}(\mathbf{q})=\left(0, \ldots, 0,-\mathbf{e}_{i j}(\mathbf{q}), 0, \ldots, 0, \mathbf{e}_{i j}(\mathbf{q}), 0, \ldots, 0\right)$ et $\mathbf{e}_{i j}(\mathbf{q})=\frac{\mathbf{q}_{j}-\mathbf{q}_{i}}{\left|\mathbf{q}_{j}-\mathbf{q}_{i}\right|}$ est le vecteur unitaire dirigé de la personne $i$ vers la personne $j$ (voir figure 1). Dès qu'il y a contact entre deux individus, la vitesse effective ne peut qu'augmenter (au sens large) la distance entre eux. Dans le modèle que nous présentons ici, la vitesse réelle $\mathbf{u}$ est définie comme la projection euclidienne de la vitesse souhaitée $\mathbf{U}$ sur l'ensemble des vitesses admissibles $C_{\mathbf{q}}$. Notre modèle s'écrit donc finalement :

$$
\left\{\begin{array}{l}
\mathbf{q}=\mathbf{q}_{0}+\int \mathbf{u} \\
\mathbf{u}=\mathrm{P}_{C_{\mathbf{q}}} \mathrm{U}
\end{array}\right.
$$

où $\mathbf{U}$ est le champ des vitesses souhaitées et $C_{\mathbf{q}}$ est défini par (1). L'étude mathématique du modèle se base sur une nouvelle formulation de (2), qui fait l'objet de la section suivante.

\section{Autre formulation et RÉsultats théoriques}

\subsection{Equation d'évolution}

On introduit le cône polaire de $C_{\mathbf{q}}$, appelé cône normal sortant :

$$
N_{\mathbf{q}}=C_{\mathbf{q}}{ }^{\circ}=\left\{\mathbf{w} \in \mathbb{R}^{2 N}, \forall \mathbf{v} \in C_{\mathbf{q}} \quad \mathbf{v} \cdot \mathbf{w} \leq 0\right\}
$$

En utilisant le lemme de Farkas (voir [2]), on peut préciser l'écriture des vecteurs de $N_{\mathbf{q}}$ :

Proposition 1.

$$
N_{\mathbf{q}}=\left\{-\sum \mu_{i j} \mathbf{G}_{i j}(\mathbf{q}), \quad \mu_{i j} \geq 0, \quad \mu_{i j}=0 \text { si } D_{i j}(\mathbf{q})>0\right\}
$$

Proposition 2. $P_{C_{\mathbf{q}}}+P_{N_{\mathbf{q}}}=I d$.

Cette propriété élémentaire, basée sur la décomposition d'un espace de Hilbert en la somme de deux cônes mutuellement polaires, est établie dans [7]. Dans notre modèle :

$$
\mathbf{u}=\mathrm{P}_{C_{\mathbf{q}}} \mathbf{U}
$$




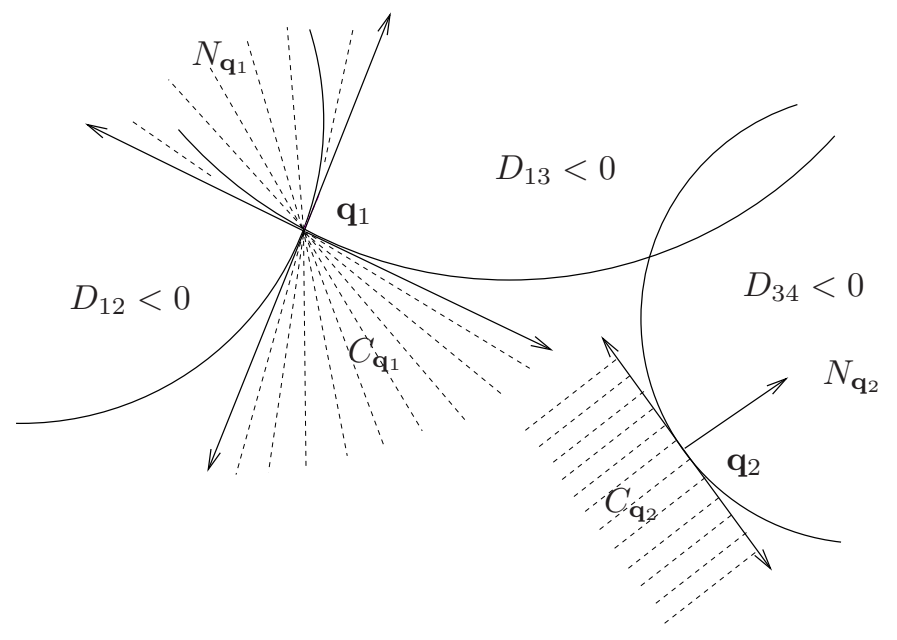

Figure 3. Cônes $C_{\mathbf{q}}$ et $N_{\mathbf{q}}$

En remplaçant $\mathrm{P}_{C_{\mathbf{q}}}$ par $I d-\mathrm{P}_{N_{\mathbf{q}}}$, on obtient :

$$
\mathbf{u}+\mathrm{P}_{N_{\mathbf{q}}} \mathbf{U}=\mathbf{U}
$$

ce qui implique que $t \mapsto \mathbf{q}(t)$ vérifie l’inclusion différentielle

$$
\dot{\mathbf{q}}+N_{\mathbf{q}} \ni \mathbf{U}(\mathbf{q})
$$

Cette inclusion a été obtenue comme conséquence du modèle initial. Nous verrons que cette formulation caractérise en fait la trajectoire $\mathbf{q}$ dans les cas qui nous intéressent.

\subsection{Résultats Théoriques}

Nous décrivons ici, sans rentrer dans les détails, le contexte théorique dans lequel s'inscrit le modèle que nous proposons.

Cas monotone. Si l'on considère le cas de personnes se déplaçant dans un couloir vide et étroit de telle sorte qu'elles effectuent un mouvement rectiligne, on peut alors écrire $Q_{0}=\left\{\mathbf{q} \in \mathbb{R}^{N}, \mathbf{q}_{i+1}-\mathbf{q}_{i} \geq r_{i+1}+r_{i}\right\}$. On impose seulement un ordre sur la position des personnes qui sera conservé grâce à la contrainte de nonchevauchement. L'ensemble des configurations admissibles $Q_{0}$ est ainsi convexe, et l'on peut écrire

$$
N_{\mathbf{q}}=\partial I_{Q_{0}}(\mathbf{q})
$$

où $\partial I_{Q_{0}}(\mathbf{q})$ est le sous-différentiel de la fonction indicatrice de $Q_{0}$. L'opérateur $\mathbf{q} \mapsto N_{\mathbf{q}}$ est alors maximal monotone. Ainsi, en appliquant les résultats issus de la théorie des opérateurs maximaux monotones [1] et en supposant le caractère lipschitzien de $\mathbf{U}$, on conclut à l'existence et à l'unicité d'une solution au problème d'inclusion différentielle (3) avec une condition initiale du type $\mathbf{q}(0)=\mathbf{q}_{0} \in Q_{0}$.

Remarque 2. Toujours en s'appuyant sur des résultats issus de [1] on peut vérifier que, dans le cas précédent, la solution de (3) est en fait solution de (2).

On trouve des résultats analogues quand on considère une personne seule dans une pièce convexe. Là encore la convexité de $Q_{0}$ et l'expression de $N_{\mathbf{q}}$ nous permettent d'arriver à la même conclusion. 
Cas général. Quand on considère de nombreuses personnes évoluant dans une salle quelconque, l'ensemble $Q_{0}$ n'est plus convexe. On peut montrer que l'opérateur $\mathbf{q} \mapsto N_{\mathbf{q}}$ n'a plus de propriétés de monotonie et la méthode précédente ne s'applique donc pas.

L'équation (3) traduit le fait que le vecteur position q, soumis à la vitesse souhaitée $\mathbf{U}$, doit évoluer tout en restant dans l'ensemble $Q_{0}$. En ce sens, notre problème s'inscrit dans le cadre des processus de rafle. Ceux-ci ont été introduits par Moreau dans les années 70 [8]. Il considérait le problème suivant : un point se trouve à l'intérieur d'un convexe fermé $C$ mobile. Quand il est attrapé par le bord de $C$ qui se déplace, il part dans la direction normale à celui-ci, comme s'il était poussé par le bord, afin de rester dans $C$. Le mouvement de ce point est alors régi par l'équation suivante :

$$
-\dot{\mathbf{q}} \in \partial I_{C(t)}(\mathbf{q}) .
$$

Celle-ci fait intervenir un opérateur maximal monotone dont le domaine dépend du temps. Pour résoudre ce problème, Moreau construit une suite de solutions discrètes selon un principe dit de rattrapage. Sous certaines hypothèses de régularité de la fonction multivaluée $t \mapsto C(t)$, l'intervalle de temps est divisé en sous-intervalles $I_{k}$ où $C(\cdot)$ varie peu. La trajectoire d'ensembles $t \mapsto C(t)$ est approchée par une multifonction constante par morceaux, valant $C_{k}$ sur $I_{k}$, ce qui permet de construire une fonction $\tilde{\mathbf{q}}$ constante sur chaque $I_{k}$ telle que $\tilde{\mathbf{q}}_{k+1}=\operatorname{Proj}\left(\tilde{\mathbf{q}}_{k}, C_{k+1}\right)$. Il démontre alors la convergence de cette suite de fonctions lorsque le pas de la subdivision tend vers zéro, obtenant à la limite une solution de (4). La régularité de la solution dépend de celle de la multifonction $C(\cdot)$. Ce schéma de preuve basé sur l'algorithme de rattrapage (catching-up) a été récemment réutilisé dans $[3,5]$ pour l'étude des processus de rafle par des ensembles non convexes. Plus précisément, ces derniers considèrent des ensembles $\mathrm{S}$, qui sont dits $\eta$-prox-réguliers, au sens suivant : il existe un réel $\eta>0$ tel que pour tout point $\tilde{\mathbf{q}}$ à distance $\mathrm{d}<\eta$ de $\mathrm{S}$, la projection de $\tilde{\mathbf{q}}$ sur $\mathrm{S}$ soit bien définie. En particulier, les ensembles convexes sont $\infty$-prox-réguliers. On peut montrer que l'ensemble $Q_{0}$ qui nous intéresse ici, est bien $\eta$-prox-régulier et $N_{\mathbf{q}}$ s'identifie bien au cône proximal normal à $Q_{0}$ en $\mathbf{q}$. Le problème d'évolution que nous considérons est ainsi couvert par les résultats de Thibault et Edmond $[4,5,9]$, qui nous permettent d'énoncer le théorème suivant :

Théorème 1. On suppose $\mathbf{q} \longmapsto \mathbf{U}(\mathbf{q})$ lipschitzien. Alors pour tout $T>0$, toute donnée initiale $\mathbf{q}_{0} \in Q_{0}$, il existe un unique $\mathbf{q} \in W^{1,1}\left(0, T ; Q_{0}\right)$ solution de

$$
\left\{\begin{array}{l}
\frac{d \mathbf{q}}{d t}+N_{\mathbf{q}} \ni \mathbf{U}(\mathbf{q}), \\
\mathbf{q}(0)=\mathbf{q}_{0} .
\end{array}\right.
$$

Remarque 3. La constante $\eta$ dépend dans notre cas du nombre de personnes $N$ et des rayons $r_{i}$ des disques qui les représentent.

\section{SCHÉmA NUMÉRIQUe}

Soit $0=t_{0}<t_{1}<\ldots<t_{p}=T$ une subdivision uniforme de l'intervalle $[0, \mathrm{~T}]$, avec $\mathrm{h}=t_{n+1}-t_{n}$, on note :

- $\mathbf{q}^{n}$ la valeur approchée de la configuration $\mathbf{q}\left(t_{n}\right)$,

- $\mathbf{u}^{n}$ la valeur approchée de la vitesse réelle $\mathbf{u}\left(t_{n}\right)$.

Connaissant $\mathbf{q}^{n}$, on obtient la configuration au temps suivant, en utilisant un schéma d'Euler explicite. Reste à déterminer la vitesse réelle $\mathbf{u}^{n}$ à partir de la vitesse souhaitée $\mathbf{U}\left(\mathbf{q}^{n}\right)$. Là encore, la première s'exprime comme 
la projection de la seconde sur un ensemble de vitesses admissibles au premier ordre.

$$
\begin{array}{ll}
\text { Initialisation : } & \mathbf{q}^{0}=\mathbf{q}_{0} \\
\text { Boucle en temps : } & \mathbf{q}^{n} \text { connu } \\
& \mathbf{u}^{n}=\mathrm{P}_{C_{h}\left(\mathbf{q}^{n}\right)}\left(\mathbf{U}\left(\mathbf{q}^{n}\right)\right) \\
& \text { où } C_{h}\left(\mathbf{q}^{n}\right)=\left\{\mathbf{v} \in \mathbb{R}^{2 N}, D_{i j}\left(\mathbf{q}^{n}\right)+\mathrm{h} \mathbf{G}_{i j}\left(\mathbf{q}^{n}\right) \cdot \mathbf{v} \geq 0 \quad \forall i<j\right\} \\
& \mathbf{q}^{n+1}=\mathbf{q}^{n}+\mathrm{h} \mathbf{u}^{n}
\end{array}
$$

Cet algorithme s'inspire d'un schéma initialement développé pour les écoulements granulaires [6]. On peut montrer que ce schéma ne fournit que des configurations admissibles.

Proposition 3. Pour tout $n$, tous $i \neq j$, on a $D_{i j}\left(\mathbf{q}^{n}\right) \geq 0$.

Proof. C'est une conséquence directe de la convexité de la fonction $\mathbf{q} \longmapsto D_{i j}(\mathbf{q})$. En effet,

$$
\begin{aligned}
D_{i j}\left(\mathbf{q}^{n+1}\right) & =D_{i j}\left(\mathbf{q}^{n}+\mathrm{h} \mathbf{u}^{n}\right) \\
& \geq D_{i j}\left(\mathbf{q}^{n}\right)+\mathrm{h} \mathbf{G}_{i j}\left(\mathbf{q}^{n}\right) \cdot \mathbf{u}^{n}\left(\text { convexité de } D_{i j}\right)
\end{aligned}
$$

Remarque 4. La vitesse réelle $\mathbf{u}^{n}$ est obtenue comme projection orthogonale de $\mathbf{U}\left(\mathbf{q}^{n}\right)$ sur un ensemble convexe fermé qui contient 0 . Les normes $\ell^{2}$ sur $\mathbb{R}^{2 N}$ vérifient donc : $\left|\mathbf{u}^{n}\right| \leq\left|\mathbf{U}\left(\mathbf{q}^{n}\right)\right|$. On prendra garde au fait qu'en revanche aucun principe du maximum n'est vérifié : le module de la vitesse d'un individu peut notamment être supérieur au module de la vitesse souhaitée. On vérifie en effet numériquement que certaines personnes peuvent être poussées et avoir une vitesse réelle plus grande en module que leur vitesse souhaitée, même si la vitesse souhaitée est de module constant.

\subsection{Calcul approché de la vitesse effective}

Dans cette section, nous allons préciser la méthode numérique adoptée pour calculer $\mathbf{u}^{n}$. Pour alléger les notations, nous supprimons ici toute référence au pas de temps courant (les exposants $n$ sont omis). La vitesse

réelle $\mathbf{u}$ est solution du problème de minimisation sous contrainte suivant : $\min _{\mathbf{v} \in C_{h}(\mathbf{q})}|\mathbf{v}-\mathbf{U}(\mathbf{q})|^{2}$. Le lagrangien du problème point-selle associé est :

$$
L(\mathbf{v}, \boldsymbol{\lambda})=\frac{1}{2}|\mathbf{v}-\mathbf{U}(\mathbf{q})|^{2}-\sum_{1 \leq i<j \leq N} \lambda_{i j}\left(D_{i j}(\mathbf{q})+\mathrm{h} \mathbf{G}_{i j}(\mathbf{q}) \cdot \mathbf{v}\right)
$$

L'existence d'un point-selle $(\mathbf{u}, \boldsymbol{\lambda})$ pour $L$ est immédiate (voir [2]). On a alors la relation :

$$
\mathbf{u}=\mathbf{U}(\mathbf{q})+\mathrm{h} \sum_{1 \leq i<j \leq N} \lambda_{i j} \mathbf{G}_{i j}(\mathbf{q})
$$

On définit les applications linéaires:

$$
\begin{aligned}
\Phi: & \mathbb{R}^{2 N} \\
\mathbf{v} & \mapsto-\mathrm{R}\left(\mathbf{G}_{i j}(\mathbf{q}) \cdot \mathbf{v}\right)_{i<j}
\end{aligned}
$$

et

$$
\begin{aligned}
& \Phi^{\star}: \quad \mathbb{R}^{\frac{N(N-1)}{2}} \rightarrow \mathbb{R}^{2 N} \\
& \boldsymbol{\lambda} \quad \mapsto \quad-\mathrm{h} \sum_{i<j} \lambda_{i j} \mathbf{G}_{i j}(\mathbf{q}) .
\end{aligned}
$$




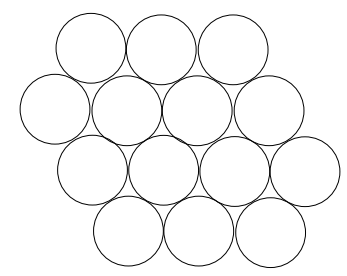

Figure 4. Cas de non-unicité du multiplicateur $\boldsymbol{\lambda}$

On peut alors réécrire l'ensemble $C_{h}(\mathbf{q})$ :

$$
\begin{aligned}
C_{h}(\mathbf{q}) & =\left\{\mathbf{v} \in \mathbb{R}^{2 N}, \forall \boldsymbol{\lambda} \in\left(\mathbb{R}^{+}\right)^{\frac{N(N-1)}{2}},-\sum_{1 \leq i<j \leq N} \lambda_{i j}\left(D_{i j}(\mathbf{q})+\mathrm{h} \mathbf{G}_{i j}(\mathbf{q}) \cdot \mathbf{v}\right) \leq 0\right\} \\
& =\left\{\mathbf{v} \in \mathbb{R}^{2 N}, \forall \boldsymbol{\lambda} \in\left(\mathbb{R}^{+}\right)^{\frac{N(N-1)}{2}},\langle\boldsymbol{\lambda}, \Phi(\mathbf{v})-\mathbf{D}(\mathbf{q})\rangle \leq 0\right\}
\end{aligned}
$$

Toutes ces notations étant fixées, précisons l'algorithme d'Uzawa qui permet de déterminer $\mathbf{u}$.

$$
\begin{aligned}
\boldsymbol{\mu}^{0} & =0 \\
\mathbf{v}^{k+1} & =\mathbf{U}(\mathbf{q})-\Phi^{\star}\left(\boldsymbol{\mu}^{k}\right) \\
\boldsymbol{\mu}^{k+1} & =\Pi_{+}\left(\boldsymbol{\mu}^{k}+\rho\left[\Phi\left(\mathbf{v}^{k+1}\right)-\mathbf{D}(\mathbf{q})\right]\right)
\end{aligned}
$$

où $\rho$ est une constante strictement positive et $\Pi_{+}$est le projecteur orthogonal sur $\left(\mathbb{R}^{+}\right)^{\frac{N(N-1)}{2}}$ :

$$
\boldsymbol{\mu} \longmapsto \Pi_{+}(\boldsymbol{\mu})=\left(\max \left(0, \mu_{i j}\right)\right)_{i<j} .
$$

La convergence de cet algorithme est assurée dès que $0<\rho<\rho_{\max }=2 /\|\Phi\|^{2}$ (voir [2]). Pour les simulations, on choisit de s'arrêter dès que le chevauchement relatif se situe en dessous d'un certain seuil.

Remarque 5. S'il y a toujours existence du multiplicateur de Lagrange pour ce problème de dimension finie, son unicité n'est pas assurée en général. Ainsi, on n'a pas unicité lorsque la disposition des personnes forme un amas cristallin assez grand. Considérons par exemple la configuration de $N=14$ personnes représentée sur la figure 4, on peut dénombrer 29 contacts actifs. Autrement dit, la dimension de l'espace où vit $\boldsymbol{\lambda}$ est 29, alors que l'espace des configurations a pour dimension : $2 \times 14=28 . L^{\prime}$ 'application $\Phi^{\star}$ n'est donc pas injective. Par conséquent, l'ensemble

$$
\Lambda=\left\{\boldsymbol{\lambda} \in\left(\mathbb{R}^{+}\right)^{\frac{N(N-1)}{2}}, \mathbf{w}=\sum_{i<j} \lambda_{i j} \mathbf{G}_{i j}(\mathbf{q})\right\}
$$

n'est pas réduit en général à un singleton. En revanche, on peut montrer que cet ensemble, quel que soit le nombre de personnes et de contacts, est borné (cf. [6]).

Remarque 6. Le nombre de contacts susceptibles d'être activés est nettement inférieur à $\frac{N(N-1)}{2}$. Dans le cas où tous les rayons sont égaux à $r$ par exemple, chaque disque est en contact avec au plus 6 autres. Le nombre de contacts est alors inférieur à $3 N$. Lors de l'implémentation, seules les contraintes correspondant à deux personnes proches sont activées.

Nous terminons par une comparaison entre l'approche théorique du problème et notre schéma numérique. Pour démontrer leurs résultats, les auteurs de $[3,5]$ ont construit une solution discrète en utilisant le fait qu'à 


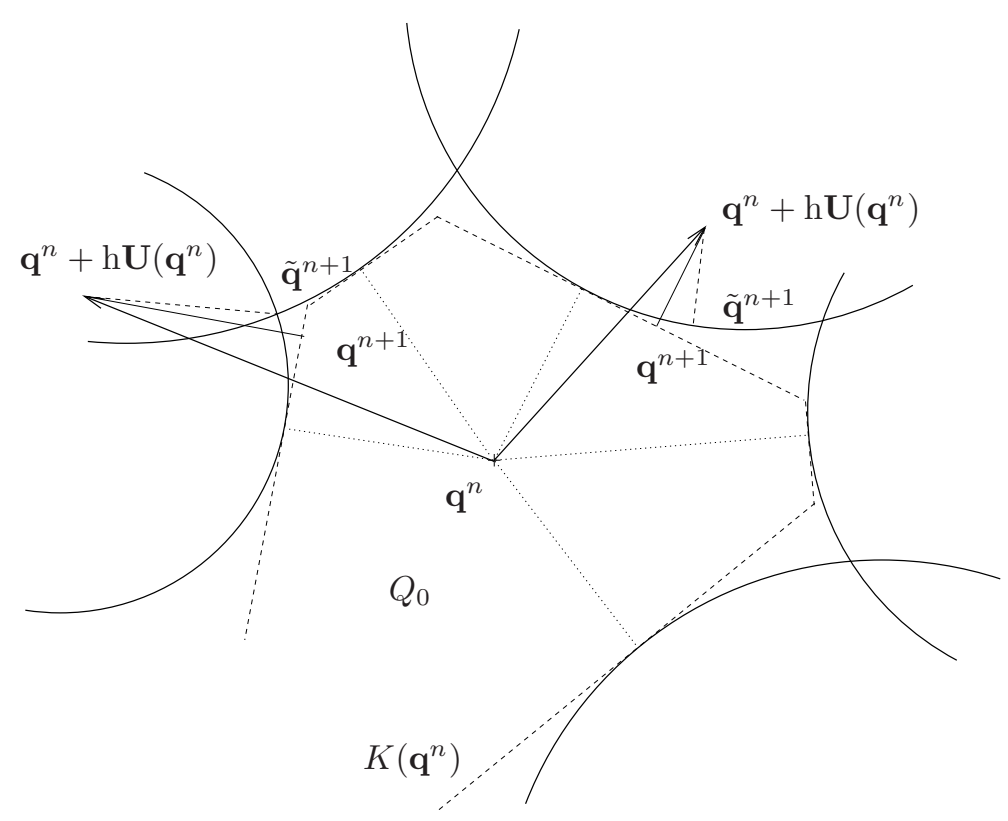

Figure 5. Projections théorique et numérique

une distance suffisamment petite de $Q_{0}$, la projection sur celui-ci était bien définie. Si l'on interprète notre algorithme en termes de position, on a:

$$
\begin{gathered}
\mathbf{q}^{n+1}=\mathrm{P}_{K\left(\mathbf{q}^{n}\right)}\left(\mathbf{q}^{n}+\mathrm{h} \mathbf{U}\left(\mathbf{q}^{n}\right)\right) \\
\text { où } K\left(\mathbf{q}^{n}\right)=\left\{\mathbf{q} \in \mathbb{R}^{2 N}, D_{i j}\left(\mathbf{q}^{n}\right)+\mathbf{G}_{i j}\left(\mathbf{q}^{n}\right) \cdot\left(\mathbf{q}-\mathbf{q}^{n}\right) \geq 0 \quad \forall i<j\right\} .
\end{gathered}
$$

On projette en fait sur $K\left(\mathbf{q}^{n}\right)$ que l'on peut qualifier d'approximation convexe intérieure de $Q_{0}$ (voir Fig. 5). À chaque pas de temps, on résout ainsi un problème sur un espace admissible strictement plus petit. Sur la figure 5 , les frontières de $K\left(\mathbf{q}^{n}\right)$ sont en pointillé. Les points $\mathbf{q}^{n+1}$ et $\tilde{\mathbf{q}}^{n+1}$ sont obtenus respectivement après projection sur $K\left(\mathbf{q}^{n}\right)$ et sur $Q_{0}$ de $\mathbf{q}^{n}+\mathrm{h} \mathbf{U}\left(\mathbf{q}^{n}\right)$ (pour les deux exemples de $\mathbf{U}\left(\mathbf{q}^{n}\right)$ donnés).

\section{Tests NumÉRIQUeS}

Les calculs présentés ci-dessous ont été effectués sous Matlab, sans souci d'optimisation du temps de calcul ou de stockage mémoire. Notre but était juste de vérifier le programme sur quelques cas simples. Dans le premier exemple, les calculs ont pris 40 minutes, dans le second $2 \mathrm{~h} 30$. La résolution du problème point-selle lors d'un important bouchon se révèle la plus coûteuse, un grand nombre d'itérations de l'algorithme d'Uzawa est nécessaire pour atteindre le seuil de précision prescrit par l'utilisateur. Les calculs lors de pas de temps sans engorgement sont quasi immédiats.

\subsection{Accès à un escalator}

Ce premier exemple traite de 300 personnes qui sortent d'un train et se dirigent vers un escalator. Elles se déplacent toutes à la même allure. Le paramètre $h$ est fixé de telle sorte qu'une personne libre de ses mouvements parcourt en un pas de temps sa propre taille. 

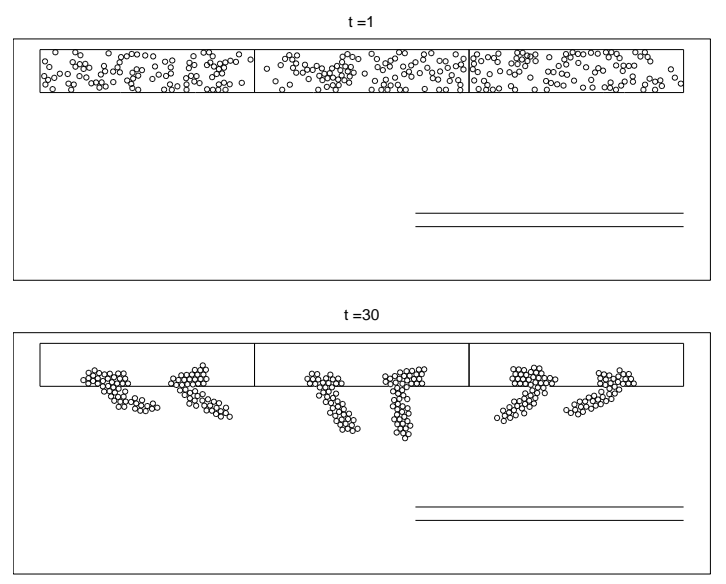

$t=120$

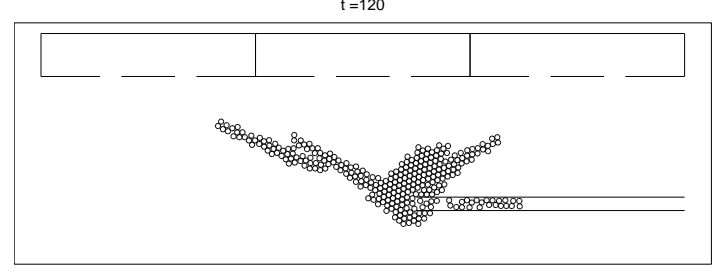

$t=400$

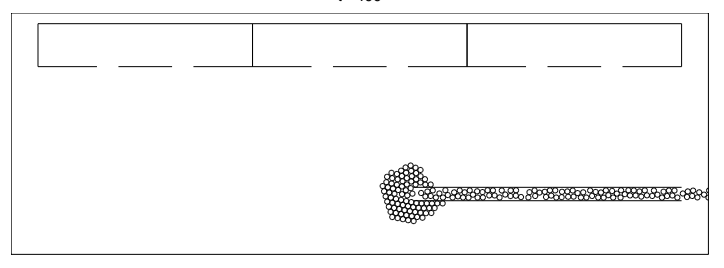

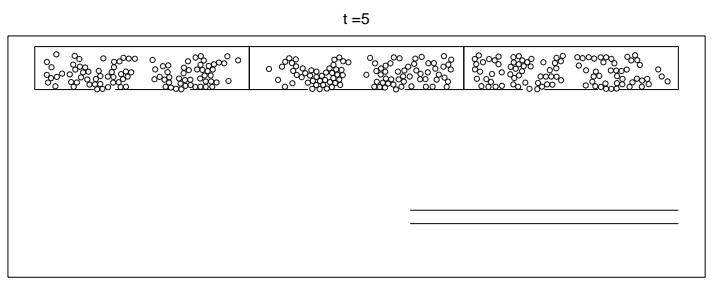

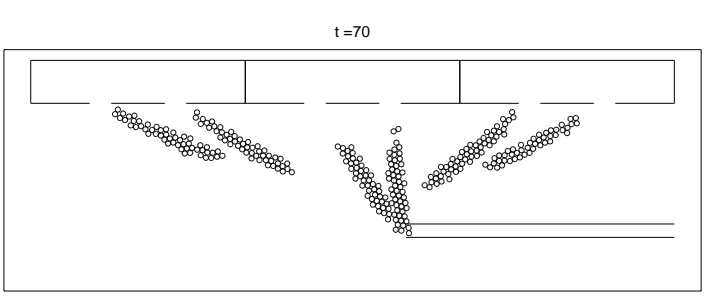

$t=210$

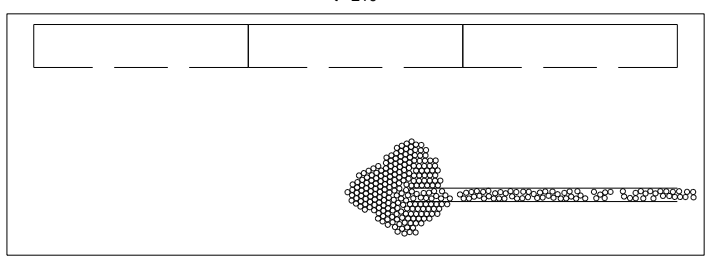

$t=540$

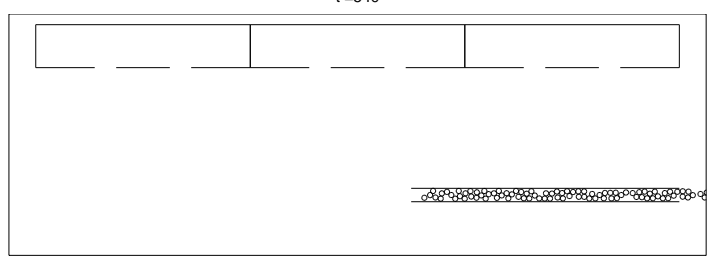

\subsection{Evacuation à deux vitesses}

Ici, il s'agit de l'évacuation d'environ 1000 personnes d'un bâtiment composé de deux salles reliées par un couloir. Le flot du champ de leurs vitesses souhaitées a été tracé sur la figure 2. Pour illustrer la souplesse du modèle, nous avons considéré une population non homogène de petits disques et de gros disques (diamètre triple de celui des petits), en supposant que les petits avancent trois fois plus vite que les autres, ou plus précisément que leur vitesse souhaitée a un module trois fois supérieur. Le pas de temps $h$ est pris de telle manière qu'un petit disque libre parcourt en un pas de temps son propre diamètre.

$t=1$

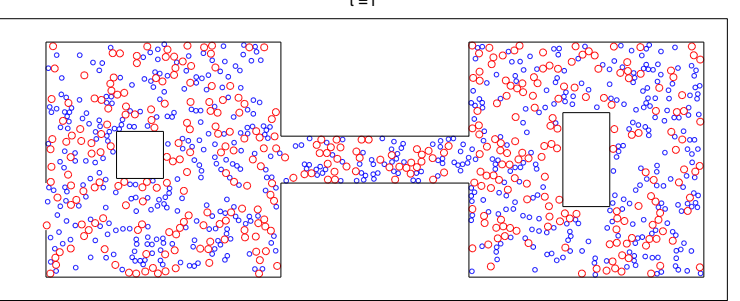

$t=20$

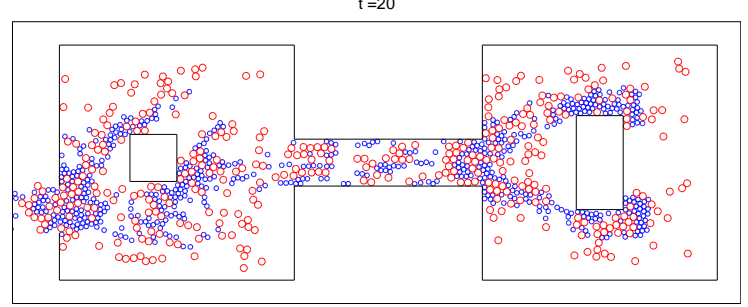



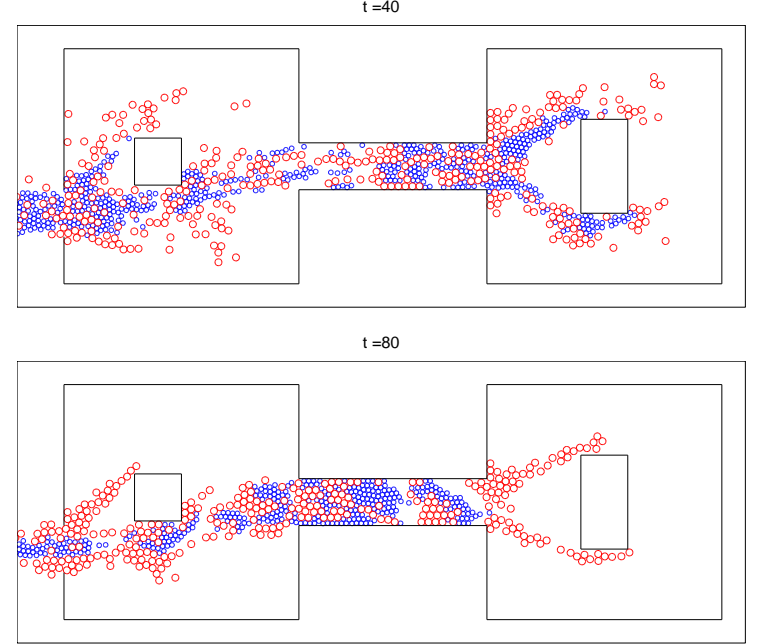

$t=120$

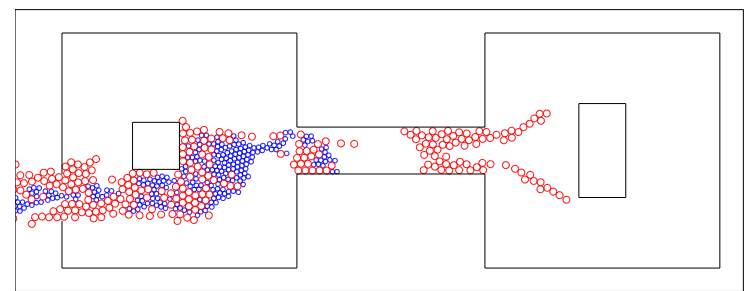

$t=60$

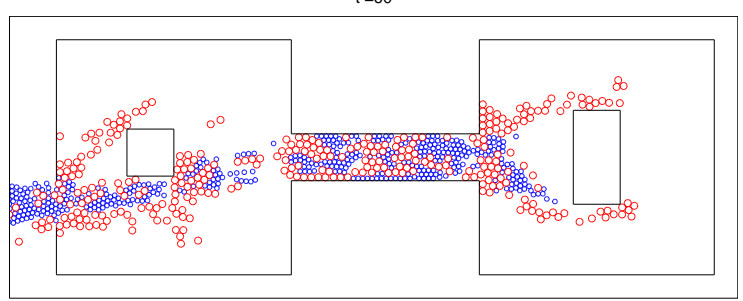

$t=100$

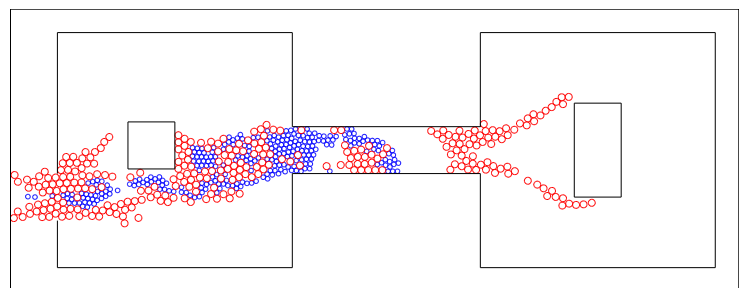

$t=140$

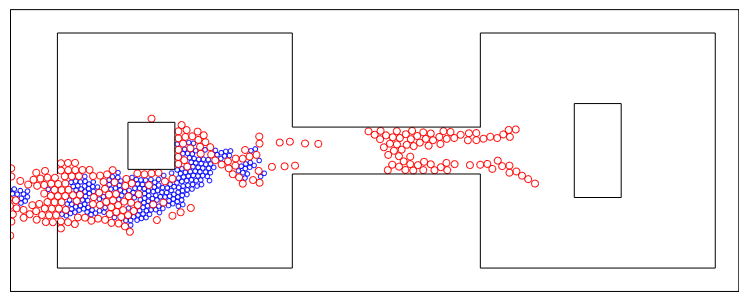

\section{REFERENCES}

[1] H. Brezis, Opérateurs Maximaux Monotones et Semi-groupes de contractions dans les espaces de Hilbert, 1973.

[2] P.G. Ciarlet, Introduction à l'analyse numérique matricielle et à l'optimisation, Masson, Paris, 1990.

[3] G. Colombo, M.D.P. Monteiro Marques, Sweeping by a continuous prox-regular set, J. Differential Equations 187, no. 1, 46-62, 2003.

[4] J.F. Edmond, L. Thibault, Relaxation of an optimal control problem involving a perturbed sweeping process, Math. Program 104, no. 2-3, Ser. B, 347-373, 2005.

[5] J.F. Edmond, L. Thibault, BV solutions of nonconvex sweeping process differential inclusion with perturbation, J. Differential Equations, à paraître.

[6] B. Maury, A time-stepping scheme for inelastic collisions, Numerische Mathematik 102, no. 4, 649-679, 2006.

[7] J.J. Moreau, Décomposition orthogonale d'un espace hilbertien selon deux cônes mutuellement polaires, C. R. Acad. Sci., Série I, 255, 238-240, 1962. 347-374, 1977

[8] J.J. Moreau, Evolution Problem associated with a moving convex set in a Hilbert space, J. Differential Equations 26, no. 3, 347-374, 1977.

[9] L. Thibault, Sweeping Process with regular and nonregular sets, J. Differential Equations 193, no. 1, 1-26, 2003. 